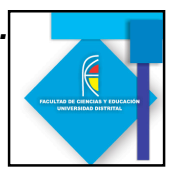

\title{
LAS RELACIONES CTSA CON LA FORMACIÓN DE MAESTROS EN CONTEXTOS DE VULNERABILIDAD
}

\section{THE RELATIONS STSE WITH THE TEACHERS' FORMATION IN CONTEXTS OF VULNERABILITY}

\author{
Fredy Gregorio Valencia Valbuena ${ }^{1}$ \\ Carmen Giraldo ${ }^{2}$
}

\begin{abstract}
Resumen
La cátedra electiva "Trabajo Pedagógico con Comunidades en Condición de Desplazamiento" adscrita al Departamento de Física, Facultad de Ciencia y Tecnología, de la Universidad Pedagógica Nacional, ha realizado la reflexión teórica y práctica frente al trabajo pedagógico con comunidades que habitan barrios periféricos del municipio de Soacha (Altos de Cazucá), lugares que evidencian la migración de la población en condición de desplazamiento (Codhes, 2009).

Lo anterior ha generado que la Universidad como institución formadora de maestros, asuma la responsabilidad ética y política con los grandes problemas de la educación colombiana, y para ello contribuye con el desarrollo de un conjunto de propuestas significativas de estudiantes de varios programas curriculares a partir del ejercicio como maestro(a) en determinados escenarios de alta vulnerabilidad social, para lo cual se propone articular las disciplinas con el contexto, en la perspectiva de la formación de ciudadanos participativos para la toma de decisiones a partir de problemáticas de su entorno que contribuyen para la búsqueda de posibles alternativas de solución a partir del planteamiento de situaciones a través del diálogo, el saber escuchar, argumentar y controvertir (Caamaño, 1995), en este sentido se articula los escenarios vulnerables como posibilitadores para el trabajo en conjunto (profesores-estudiantes-comunidad) desde los aportes del enfoque ciencia, tecnología, sociedad y ambiente (CTSA).
\end{abstract}

Palabras Clave: Vulnerabilidad, CTSA, formación de maestros.

\begin{abstract}
The elective courses "Teaching Working with Communities in displacement condition" attached to the Department of Physics, Faculty of Science and technology, the Universidad Pedagógica Nacional, has done the theoretical and practical, the reflexion practical pedagogical work with the communities living in the suburbs of Soacha (Altos

${ }^{1}$ Docente Centro Integral "José María Córdoba" I.E.D. Profesor Catedrático del Departamento de Física, fvalencia@pedagogica.edu.co

${ }_{2}^{2}$ Profesora Catedrática del Departamento de Física -Universidad Pedagógica Nacional, clgm940@yahoo.com
\end{abstract}




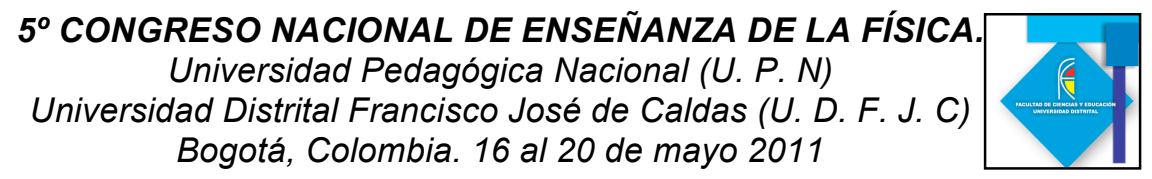

de Cazucá), places that show the migration of the population living in displacement conditions (Codhes, 2009).

This has led to the University as an institution to prepare teachers to assume the ethical and political responsibility with the big problems of education in Colombia, and for this it contributes to the development of a set of meaningful Offers of students from various curricula programs beginning with the role of the teacher in certain contexts of extreme social vulnerability, for which it is proposed to link the disciplines with the context, in view of the formation of participatory for making desizion based on the problems from their environment that contribute to the search for alternative solution from the approach of situations through dialogue, listening, arguing and being controversia (Caamaño, 1995), in this sense it is articulated vulnerable scenarios as enablers to work together (teachers-students-community) focus on contributions of the science, technology, society and environment (CTSA).

Keywords: Vulnerability, CTSA, teacher training.

\section{Introducción}

El siguiente ensayo presenta y justifica la cátedra "Trabajo Pedagógico con Comunidades en Condición de Desplazamiento" adscrita al Departamento de Física, Universidad Pedagógica Nacional, en donde estudiantes de diferentes programas de la Universidad desarrollan un trabajo interdisciplinario que posibilita la construcción de proyectos de aula significativos y pertinentes a la realidad social y cultural de los niños y niñas que se encuentran en condiciones de vulnerabilidad (desplazamiento) que habitan la comuna cuatro (Altos de Cazucá) ubicada en el municipio de Soacha (C/marca). En primer lugar se presenta la cátedra, luego se realiza una breve reflexión en torno al fenómeno del desplazamiento desde la mirada social y educativa en particular con las migraciones de las poblaciones hacia Soacha (C/marca), seguidamente se relaciona el enfoque Ciencia, Tecnología, Sociedad y Ambiente (CTSA), posteriormente se plantea la metodología y finalmente están las conclusiones.

\section{Antecedentes}

La experiencia de trabajo pedagógico desarrollado durante los últimos 6 años en el campo de la enseñanza de las ciencias, en Altos de Cazucá particularmente en las escuelas comunitarias desde la cátedra electiva "Trabajo Pedagógico con Poblaciones en Condición de Desplazamiento" ha posibilitado en dos miradas la construcción y enriquecimiento pedagógico, en primer lugar tanto para los niños y niñas que asisten a estos espacios alternativos de educación popular y en segundo lugar para la sensibilización y cualificación de los profesores en formación de la universidad, a través de la construcción de ambientes pedagógicos que permiten el desarrollo de pensamiento crítico en (niños, niñas, profesores en formación y comunidad) con la intensión de construir la concepción de ciencia contextualizada.

Se habla de niños y niñas vulnerables porque no acceden fácilmente al sistema educativo (MEN, 2004), por diferentes situaciones sociales tales como: el desplazamiento por el conflicto armado, la pobreza, el trabajo infantil y otros problemas sociales y económicos. 


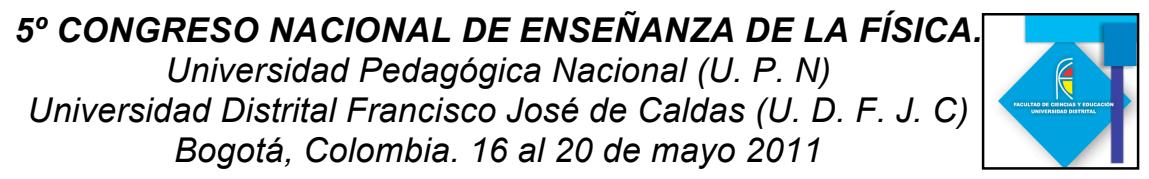

Cada niño y niña tienen derecho de saber y saber hacer (MEN, 2004) lo mismo que sus iguales, sin importar su condición social, ubicación geográfica, raza o contexto de existencia. Por lo tanto es indispensable que los niños y las niñas en condición de vulnerabilidad que habitan en la comuna de Altos de Cazucá (Soacha, C/marca) accedan a la ciencia y desde allí puedan fortalecer la escuela hacia la formación de ciudadanos participativos para la toma de decisiones a partir de problemáticas de su entorno que contribuyen para la búsqueda de posibles alternativas de solución y permita la enseñanza contextual de las ciencias en la medida de aportar posibles soluciones a su difícil situación social desde la mirada disciplinar, tecnológica, social y ambiental.

\section{Cátedra Electiva "Trabajo Pedagógico con Comunidades en Condición de Desplazamiento"}

La Universidad Pedagógica Nacional (UPN) como institución pública formadora de futuros maestros, tiene una gran responsabilidad ética y política con los grandes problemas de la educación colombiana, particularmente a la educación de niños, niñas y jóvenes en condición de vulnerabilidad, situación que a su vez involucra a éstos en otro tipo de exclusiones como la escolar. Y por otra parte, la posibilidad de concretar en la realidad la misión que se expresa en el PEI de la UPN con respecto al desarrollo y promoción de estrategias encaminadas a compensar la deuda social existente en dicho campo y las profundas desigualdades socioculturales presentes.Por lo anterior se consolida en el año 2004 la Cátedra "Trabajo Pedagógico con Comunidades en Condición de Desplazamiento" bajo la orientación de la profesora Clara Chaparro Susa.

La intensión de la cátedra corresponde particularmente con:

Construir un espacio académico que permita la cualificación disciplinar de los profesores(as) en formación de cualquier programa de la UPN además de iniciar un proceso de sensibilización frente al fenómeno del desplazamiento.

Contribuir desde los seminarios a la reflexión sobre propuestas pertinentes para la educación en comunidades que se encuentran en condición de desplazamiento.

\section{Desplazamiento en Bogotá y Soacha, el Interés por Altos de Cazucá}

Al respecto el SISDHES (Codhes, 2009) argumenta que durante el periodo de 1985 a 2008 en Colombia se desplazaron 4.629 .190 personas quienes conforman aproximadamente (925.838 familias) es decir el $17 \%$ de la población colombiana pero el gobierno registra oficialmente en su sistema de información 2.977.209 personas desplazadas para el periodo comprendido entre 1997 y marzo de 2009, en este sentido durante el 2007 se desplazaron 305.966 personas y en el 2008 se agudizo la crisis con 380.863 personas (76.172 núcleos familiares) es decir aumento el $24.47 \%$, de estos datos el $52 \%$ corresponde a población de niños, adolescentes y mujeres (Ver figura 1). 

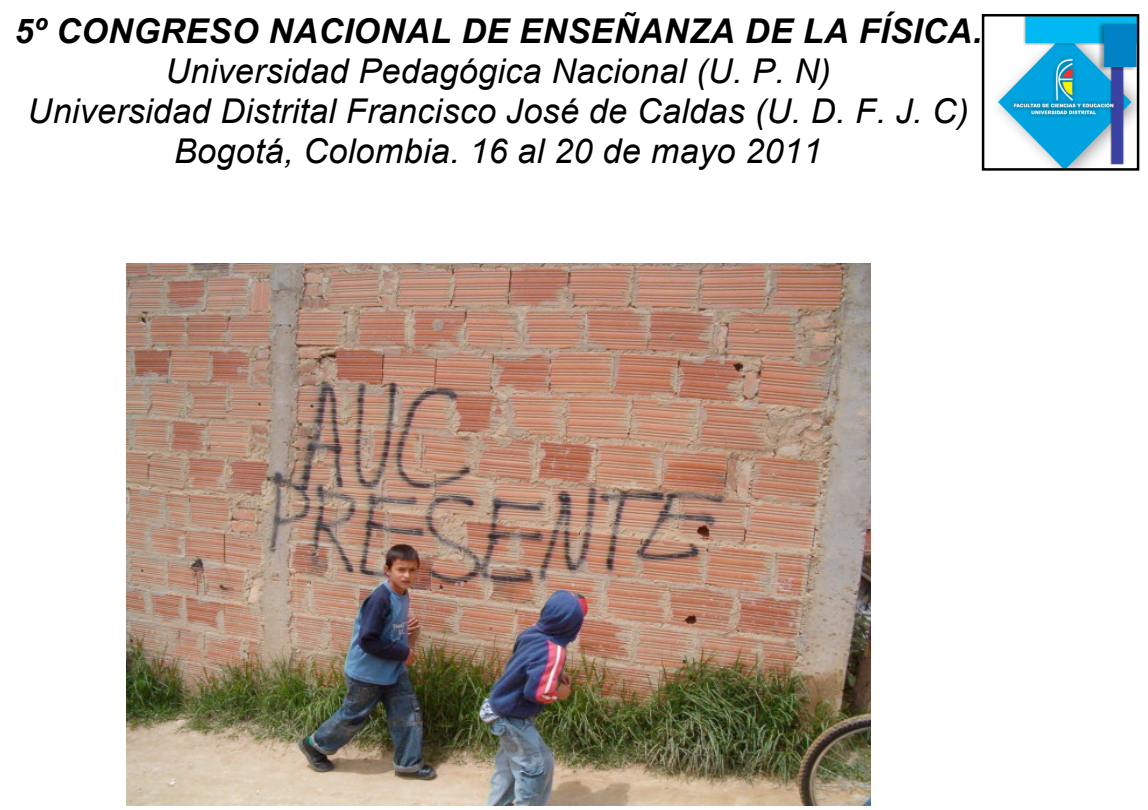

Figura 1.

El conflicto armado y la educación, tomando como referencia el numero de personas desplazadas dureante el 2008, los lugares de mayor recepción son Bogotá (56.087 personas desplazadas),Antioquia (52.276), Valle del Cauca (31.527), Magdalena (27.256), Nariño (24.662); Meta (16.370), Cauca (16.344) y Córdoba (12.879) (Codhes, 2009). En este sentido se promulga la sentencia T-025 del 2004, la cual corrobora que los derechos de la población desplazada siguen sin ser atendidos de forma integral. Los anteriores planteamientos posibilitan generar controversias entre distintos actores, en particular desde la academia es importante plantear propuestas que permitan entender y asumir la responsabilidad educativa, social y política que permita a la comunidad y en particular a la escuela apropiarse como sujetos de conocimiento (Segura, 2000) y ser entes transformadores alternativos de la realidad, con el respeto y la atención de la diversidad étnica y cultural para construir una sociedad más equitativa, pluralista, libre y defensora de los derechos humanos, en donde sea inaceptable la uniformidad y homogeneidad de las culturas (Ley 387/1997), siendo necesario viabilizar otras maneras de educación alternativas. En cuanto a la educación la corte constitucional reconoce que el acceso y permanencia de los niños, niñas y jóvenes desplazados en el sistema educativo es del $36 \%$, lo que nuevamente vislumbra la necesidad del trabajo educativo con la población desplazada (Romero, 2007).

\section{Enlazando el Enfoque Ciencia, Tecnología, Sociedad y Ambiente (CTSA) Integrando a los Profesores en Formación-Escuela-Comunidad}

Una alternativa metodológica para comprender el papel que juega la ciencia hoy día, es a través de la formación de ciudadanos críticos (niños, niñas, profesores en formación y comunidad)) que para nuestro caso corresponde con los aportes del enfoque Ciencia, Tecnología, Sociedad y Ambiente (CTSA), en particular Solbes y Vilches (1989), plantean el posibilitar una enseñanza contextualizada y pertinente a los intereses de los estudiantes a partir de las discusiones de grupo centradas a través del aprendizaje cooperativo, así mismo Caamaño (1995), pretende enseñar las ciencias en concordancia con el 


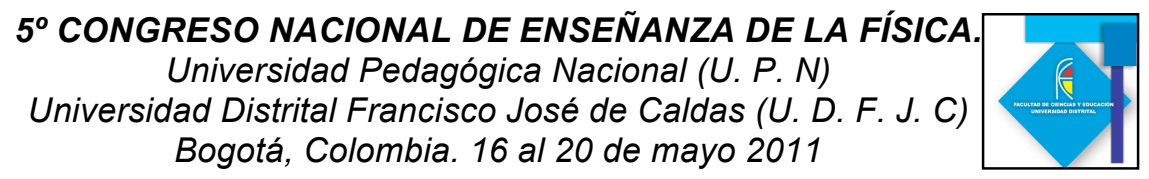

planteamiento de situaciones reales que a través del diálogo, el saber escuchar, argumentar y controvertir con argumentos sólidos permitan actuar consecuentemente.

En este sentido la cátedra le apuesta a que los profesores en formación contribuyan desde el marco de referencia del enfoque CTSA para el diálogo mutuo bidireccional para la construcción de propuestas pedagógicas que contribuyan para la enseñanza de las ciencias (Membiela, 1996) teniendo en cuenta las difíciles situaciones, sociales, culturales, políticas, ambientales y otras que son propias a las comunidades vulnerables, en particular las que habitan en Altos de Cazucá (Soacha, C/marca). En este sentido Solbes y Vilches (1989), plantean que la propuesta CTSA puede posibilitar una enseñanza contextualizada y pertinente a los intereses de los estudiantes a partir de las discusiones de grupo centradas a través del aprendizaje cooperativo desde el cual se puede asumir posiciones y roles desde los diferentes entes del Estado en cuyo procesos articulan posturas y encaminan posibles alternativas de solución para los contenidos en los que el enfoque CTSA están siendo el eje central para evidenciar la relación de la ciencia, tecnología, sociedad y ambiente.

\section{Metodología}

El trabajo realizado con el grupo de (niños, niñas, profesores en formación y comunidad) es de carácter descriptivo, interpretativo y explicativo, a través del método de análisis cualitativos (Cardona, 2002; Hernández, Fernández y Baptista, 2006) a partir de la metodología de investigación-acción (I-A), en la cual se presenta la planeación-reflexiónobservación y acción como procesos dinámicos en espiral que contribuye de manera dinamizadora a encaminar y reorientar los presupuestos investigativos que en lo teórico y lo práctico establezcan objetivos comunes. En este sentido se proponen dos momentos de acción: En el primer momento se realiza un acercamiento al contexto social y educativo de los niños y niñas de la escuela comunitaria "Corporación Fe y Esperanza" y se espera tener una aproximación al contexto, la escuela y los actores de ella, para que así pueda generarse un trabajo contextualizado en la enseñanza de las ciencias a partir de la realidad de estos sectores vulnerables, integrando el enfoque CTSA para que viabilice el desarrollo de propuestas pedagógicas que constituyen el segundo momento de la investigación.

\section{Conclusiones}

Realizar las prácticas pedagógicas con los profesores en formación de las diferentes disciplinas de las instituciones de educación superior en las comunidades y poblaciones que se encuentran en condiciones de vulnerabilidad, permite construir alternativas de trabajo pedagógico fundamentadas en la comprensión de la realidad desde la perspectiva de la investigación acción (IA).

El enfoque CTSA permite la elaboración de propuestas pedagógicas que rompen la linealidad de la enseñanza de las ciencias y posibilita reconocer a (niños, niñas, profesores en formación y comunidad) como ciudadanos participativos. 


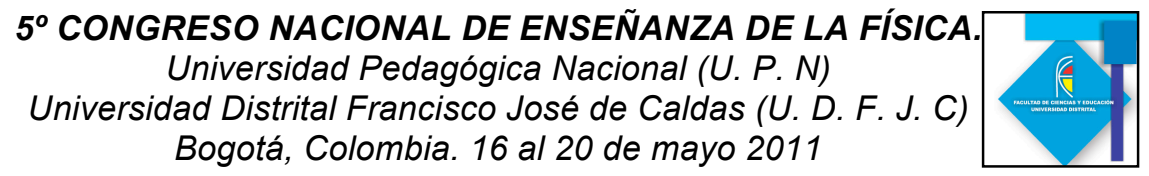

\section{Referencias Bibliográficas.}

Caamaño, A. (1995, enero). La educación CTS: una necesidad en el diseño del nuevo currículum de Ciencias. En Revista Alambique, 3, 4-6.

Cardona, M. (2002). Introducción a los Métodos de Investigación en Educación. España: Editorial EOS.

Codhes. (2009). Boletín informativo no 75. Victimas emergentes. Desplazamiento, derechos humanos y conflicto armado 2008.

Hernández, S., Fernández, C y Baptista, L. (2006). Metodología de la investigación (4ta ed.). México: McGraw Hill.

Ley 387/18 de julio de 1997. Medidas para la prevención del desplazamiento forzado. (ACNUR) Alto Comisionado de las Naciones Unidad para los Refugiados. Red de Solidaridad Social.

Membiela, P. (1996). Una revisión del movimiento educativo Ciencia-Tecnología-Sociedad. En Revista Enseñanza de las Ciencias, 15(1), 51-57.

Ministerio de Educación Nacional (MEN). (2004). Periódico Altablero. No 28 (marzo-abril). Romero, M. (2007). El desplazamiento forzado en Colombia/ 10 años de Políticas Públicas. Bogotá.

Segura, D. (2000). ¿Es posible pensar otra escuela? Colombia. EPE.

Solbes, J y Vilches, A. (1989). Interacciones Ciencia-Tecnología-Sociedad: Un instrumento de cambio actitudinal. En Revista Enseñanza de las Ciencias. 7(1), 14-20. 